\title{
Model Calculations Relevant to Disulfide Bond Cleavage via Electron Capture Influenced by Positively Charged Groups
}

\author{
Agnieszka Sawicka, ${ }^{\dagger, \$}$ Piotr Skurski, ${ }^{\dagger, *}$ Robert R. Hudgins, ${ }^{\S}$ and Jack Simons ${ }^{*, \dagger}$ \\ Department of Chemistry, Henry Eyring Center for Theoretical Chemistry, University of Utah, \\ Salt Lake City, Utah 84112, Department of Chemistry, University of Gdansk, Sobieskiego 18, \\ 80-952 Gdansk, Poland, and Department of Chemistry, York University, Toronto, Ontario, Canada
}

Received: June 12, 2003; In Final Form: September 23, 2003

\begin{abstract}
$\mathrm{Ab}$ initio electronic structure calculations are used to explore the effect of nonneighboring positively charged groups on the ability of low-energy $(<1 \mathrm{eV})$ electrons to directly attach to $\mathrm{S}-\mathrm{S} \sigma$ bonds in disulfides to effect bond cleavage. It is shown that, although direct vertical attachment to the $\sigma^{*}$ orbital of an $\mathrm{S}-\mathrm{S} \sigma$ bond is endothermic, the stabilizing Coulomb potential produced in the region of the $\mathrm{S}-\mathrm{S}$ bond by one or more distant positive groups can render the $\mathrm{S}-\mathrm{S} \sigma^{*}$ anion state electronically stable. This stabilization, in turn, can make near vertical electron attachment exothermic. The focus of these model studies is to elucidate a proposed mechanism for bond rupture that may, in addition to other mechanisms, be operative in electron capture dissociation (ECD) experiments. The importance of these findings lies in the fact that a more complete understanding of how ECD takes place will allow workers to better interpret ECD fragmentation patterns observed in mass spectrometric studies of proteins and polypeptides.
\end{abstract}

\section{Introduction}

It has been observed that disulfide linkages in proteins and peptides containing such bonds are most likely to be ruptured when low-energy electrons attach to effect fragmentation ${ }^{1}$ in electron-capture dissociation (ECD) mass spectrometric experiments. Although breaking the weakest $(\mathrm{S}-\mathrm{S})$ bond is not surprising, the mechanism by which electron capture induces the bond rupture has not been fully characterized. In such ECD samples, the molecule to which the electron attaches is positively charged and has more than one positive site. In such species, it has been suggested that electron capture can occur at protonated sites to form a hypervalent radical that subsequently induces very specific fragmentation, as shown in Scheme 1.

That is, an electron is attracted by Coulomb forces toward the protonated site (I) and attaches to a diffuse Rydberg-like orbital $^{2}$ to form the hypervalent radical (II). Subsequently, an $\mathrm{H}$ atom is released ${ }^{3}$ from the hypervalent site and a fraction of such $\mathrm{H}$ atoms ejected toward a nearby carbonyl group attack the oxygen atom of that group (carbonyl groups are known to be susceptible to such $\mathrm{H}$ atom attack) to form a carbon-centered radical species (III). Finally, rearrangement of that radical leads to $\mathrm{N}-\mathrm{C}$ bond cleavage and the formation of fragments commonly labeled $\mathrm{c}$ and $\mathrm{z}$, the latter of which typically retains the radical center. It is through the observation of characteristic $c$ and $\mathrm{z}$ fragments that ECD is used to probe protein primary structures. Indeed, recent experimental data ${ }^{1,4,5}$ and several excellent theoretical studies ${ }^{5}$ suggest the mechanism detailed in Scheme 1 as a dominant pathway by which fragmentation patterns arising in ECD experiments can be interpreted.

It has also been suggested that $\mathrm{S}-\mathrm{S}$ bond cleavage in proteins and peptides containing such disulfide bonds might proceed

\footnotetext{
* Corresponding author. E-mail: simons@chemistry.utah.edu.

$\dagger$ University of Utah.

$\doteqdot$ University of Gdansk.

$\S$ York University.
}

SCHEME 1

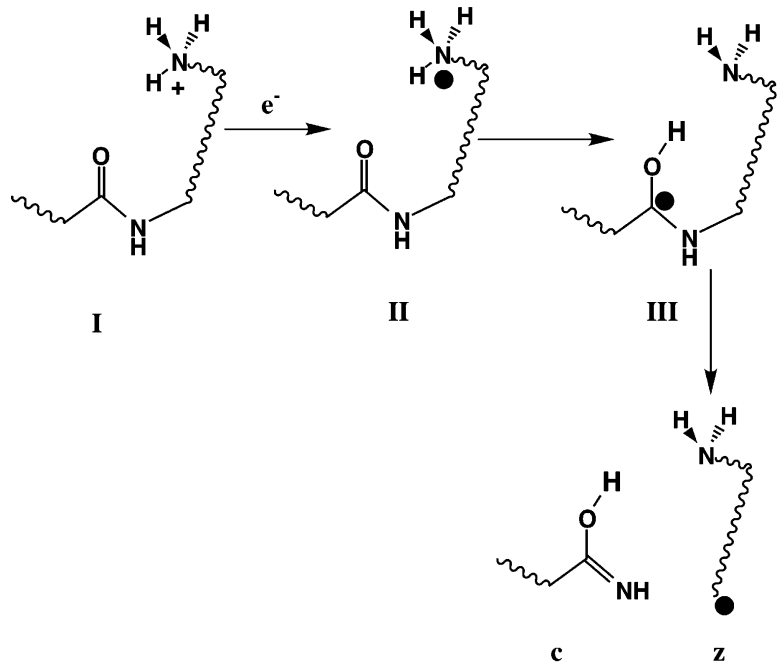

through an analogous electron capture and $\mathrm{H}$ atom migration process similar to that discussed above as illustrated in Scheme 2.

In this scheme, the $\mathrm{H}$ atom migrates after leaving the nascent $\mathrm{R}-\mathrm{NH}_{3}$ radical site and eventually makes its way to the neighborhood of the disulfide bond, which has been shown ${ }^{1,4,6}$ to be a site of high $\mathrm{H}$-atom affinity. Once near the $\mathrm{S}-\mathrm{S}$ bond, the $\mathrm{H}$ atom attacks this bond and effects $\mathrm{S}-\mathrm{S}$ bond fragmentation to produce an $\mathrm{R}-\mathrm{S} \bullet$ radical and $\mathrm{H}-\mathrm{S}-\mathrm{R}^{\prime}$. Alternatively, it has also been suggested ${ }^{4}$ that if, after electron attachment to the $\mathrm{R}-\mathrm{NH}_{3}{ }^{+}$site, a (likely solvated) proton migrates close to the $\mathrm{S}-\mathrm{S}$ bond, the presence of the latter positive charge may allow the $\mathrm{R}-\mathrm{NH}_{3}$ group's attached electron to transfer to the partially protonated $\mathrm{S}-\mathrm{S}$ bond region, thus cleaving the bond and generating $\mathrm{R}-\mathrm{S}^{\bullet}$ and $\mathrm{HS}-\mathrm{R}^{\prime}$. In either case (i.e., $\mathrm{H}$ atom migration or proton-mediated electron transfer), one would 


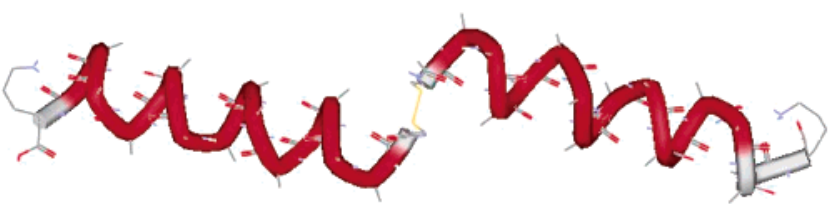

Figure 1. Structure of an $\left(\mathrm{AcCA}_{15} \mathrm{~K}+\mathrm{H}\right)_{2}{ }^{2+}$ disulfide-linked dimer from ref 8 . The disulfide linkage is at the center and the two protonated sites are at the left and right ends.

\section{SCHEME 2}

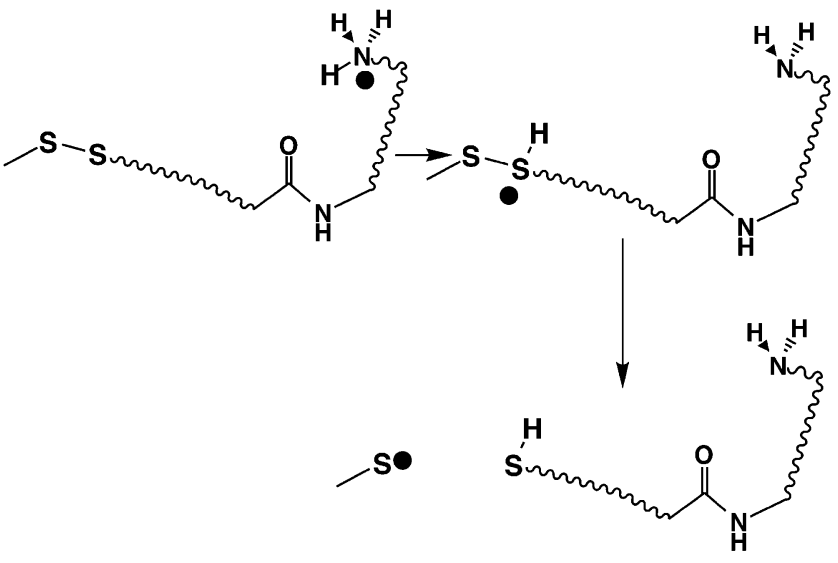

expect the efficiency of $\mathrm{S}-\mathrm{S}$ bond cleavage to decrease as the distance between the disulfide bond and the $-\mathrm{NH}_{3}$ site containing the attached electron increases.

However, recent experimental studies ${ }^{7,8}$ on $\mathrm{S}-\mathrm{S}$ bond containing synthetic peptides charged with alkali cations instead of protons also showed significant fragmentation of the $S-S$ bond, so it may be that transfer of a $\mathrm{H}$ atom from a hypervalent site to the $\mathrm{S}-\mathrm{S}$ bond is not necessary. In another recent study, ${ }^{7,8}$ disulfide-linked dimers of Ac-Cys-Ala ${ }_{n}$-Lys (with $n=10,15$, and 20) protonated at the Lys sites were used to study how the $\mathrm{S}-\mathrm{S}$ cleavage yields varied with the distance from the protonated sites to the $\mathrm{S}-\mathrm{S}$ bond. Because the $\mathrm{Ala}_{n}$ units are expected to form a helical quasilinear structure, as shown in Figure 1, we expect that the distance between the two protonated sites grows monotonically with $n$. However, in the study of ref 8 , the yield of $\mathrm{S}-\mathrm{S}$ cleavage was observed not to significantly decay, even for the $n=20$ dimer, in which the $\mathrm{S}-\mathrm{S}$ bond is thought to be ca. $32 \AA$ from either protonated site.

It should also be mentioned that the density of doubly charged cations in the ICR cell is sufficiently low that it is safe to ignore the possibility that an $\mathrm{H}$ atom ejected from one molecule attacks the $\mathrm{S}-\mathrm{S}$ bond of a different molecule. In summary, the above findings suggest that there may be another mechanism operative in ECD fragmentation, and it is this possibility that we address in this paper. We emphasize that the data discussed above and the analysis we put forth here do not suggest that the conventional Schemes 1 and 2 are not operative; rather, we argue that an additional mechanism may also be operative in ECD fragmentation.

Specifically, following the suggestion made in ref 7, we examine the possibility that low-energy electrons may also attach directly to $\mathrm{S}-\mathrm{S}$ bonds to effect fragmentation. Because even low-energy electrons have high speeds (e.g., a $0.1 \mathrm{eV}$ electron moves ca. $10^{15} \AA \mathrm{s}^{-1}$ ), the duration of their close interaction with any chemical bond is very short. As a result, any excitation or other change they effect occurs in a "vertical" manner; that is, the molecule's internal geometry remains essentially unchanged. Moreover, because they are much lighter than the molecule's nuclei, it is very inefficient for electrons to transfer
SCHEME 3

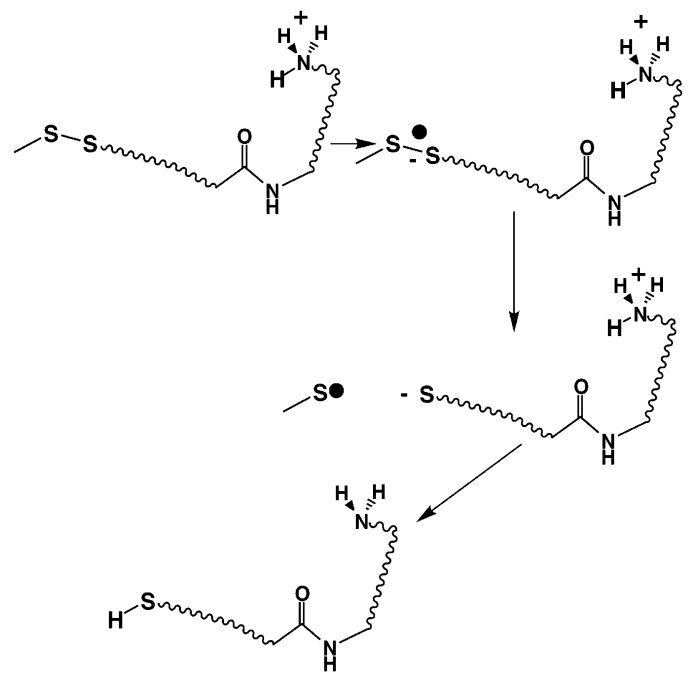

momentum and kinetic energy via impulsive collisions with nuclei. Instead, it is most common for electrons to induce electronic excitation or ionization or to attach to the molecule. Because ECD electrons have very low energies, they are not able to cause ionization of the molecule and are able to induce only very low energy electronic excitations. However, as we discuss in this paper, such low-energy electrons can induce major structural changes by attaching to vacant orbitals such as the $\mathrm{S}-\mathrm{S} \sigma^{*}$ orbitals.

The problem with suggesting that disulfide bonds can directly attach electrons in ECD experiments is that the $\sigma^{*}$ orbital into which the electron must attach is known to lie ca. $1 \mathrm{eV}$ above the energy of the neutral $\mathrm{R}-\mathrm{S}-\mathrm{S}-\mathrm{R}^{\prime}$ species at the equilibrium $\mathrm{S}-\mathrm{S}$ bond length appropriate to vertical electron attachement. However, the ECD source ${ }^{9}$ does not have many electrons with such high kinetic energy. Specifically, electron transmission spectroscopy (ETS) experiments ${ }^{10}$ on $\mathrm{Me}_{2} \mathrm{~S}_{2}$ clearly show the $\mathrm{S}-\mathrm{S} \sigma^{*} \mathrm{Me}_{2} \mathrm{~S}_{2}{ }^{-}$shape resonance state to lie $1.04 \mathrm{eV}$ above the neutral $\mathrm{Me}_{2} \mathrm{~S}_{2}$ and to have a half-width of ca. $0.5 \mathrm{eV}$. This Heisenberg width suggests an autodetachment lifetime of $10^{-14}$ $\mathrm{s}$, so the $\mathrm{S}-\mathrm{S}$ bond has little time to move prior to autodetachment of the electron. This same work shows the $\mathrm{S}-\mathrm{C} \sigma^{*}$ state in $\mathrm{Me}_{2} \mathrm{~S}_{2}$ to lie at $2.7 \mathrm{eV}$ at the equilibrium $\mathrm{C}-\mathrm{S}$ bond length.

Recent Rydberg electron transfer (RET) experiments, which are designed to probe the adiabatic rather than vertical electron attachment process, show ${ }^{11}$ that $\mathrm{MeS}-\mathrm{SMe}^{-}$at its equilibrium $\mathrm{S}-\mathrm{S}$ distance lies ca. $0.1 \mathrm{eV}$ below $\mathrm{MeS}-\mathrm{SMe}$ at its optimal $\mathrm{S}-\mathrm{S}$ distance. However, unlike the RET experiments, where the electron is thought to transfer in an adiabatic manner in which the nuclei have time to move, the ECD electron attachment process occurs in a more nearly vertical manner. Hence, the above experimental data suggest it is improbable that ECD electrons attach directly (i.e., vertically) to $S-S$ bonds and persist long enough for the $\mathrm{S}-\mathrm{S}$ bond to move to longer distances and rupture in the absence of any effect that can alter the energy of the $\sigma^{*}$ anion.

The alternative mechanism we explore here involves directly attaching a low-energy electron to an $\mathrm{S}-\mathrm{S}$ bond whose $\sigma^{*}$ anion state has been lowered in energy (relative to the neutral) by the Coulomb stabilization potential produced by distant positively charged sites elsewhere in the molecule. For example, as we show in Scheme 3, this initially generates a $\mathrm{R}-\mathrm{S} \bullet$ radical and $\mathrm{a}^{-} \mathrm{S}-\mathrm{R}-\mathrm{NH}_{3}{ }^{+}$ion pair ${ }^{12}$ that contains the protonated site; the latter could subsequently undergo proton transfer to generate $\mathrm{HS}-\mathrm{R}-\mathrm{NH}_{2}$. Note that the final products of Scheme 3 are 
exactly the same as in Scheme 2 and are consistent with what is found experimentally.

In invoking the process outlined above, we posit that the $\sigma^{*}$ anion's energy $E$ relative to the energy of the neutral at its equilibrium $\mathrm{S}-\mathrm{S}$ bond length can be approximated by lowering the intrinsic ca. $1 \mathrm{eV}$ instability of the anion state by the sum of Coulomb stabilizations due to positive sites in the molecule

$$
E=1 \mathrm{eV}-\sum_{J} 14.4 \mathrm{eV} \mathrm{R}_{J}^{-1}
$$

where $R_{J}$ is the distance from the $J^{\text {th }}$ positive site to the midpoint of the $\mathrm{S}-\mathrm{S}$ bond in $\AA$. In the ab initio quantum calculations that we describe below, we examined a series of model systems designed to test this hypothesis. It should be noted that for the kind of synthetic peptide shown in Figure 1, there are also local dipoles within the $n$ alanine groups that can also contribute to the total electrostatic potential in the region of the $S-S$ bond. In the present study, we have not tried ${ }^{13}$ to include in our model such dipolar potentials, but we do intend to examine them in future simulations.

\section{Methods}

The equilibrium geometries of the neutral $\mathrm{MeS}-\mathrm{SMe}$ and anionic $\mathrm{MeS}-\mathrm{SMe}^{-}$and their harmonic vibrational frequencies were calculated at the unrestricted second-order Møller-Plesset (UMP2) perturbation level of theory with aug-cc-pVDZ basis sets. ${ }^{14}$ In addition, partial MP2 geometry optimization (i.e., with only the $\mathrm{S}-\mathrm{S}$ bond distance frozen) calculations were employed to achieve the energies for relaxed scans of the potential energy surfaces of $\mathrm{MeS}-\mathrm{SMe}$ and $\mathrm{MeS}-\mathrm{SMe}^{-}$as functions of the $\mathrm{S}-\mathrm{S}$ distance.

In all calculations, the values of $\left\langle S^{2}\right\rangle$ never exceeded 0.7511 (after annihilation) for the doublet states, so we are confident that spin-contamination effects are negligible. To examine the effects of basis-set superposition errors (BSSE), we used the procedure described in ref 15 . The noncorrected relaxed scans for the neutral and anionic $\mathrm{MeS}-\mathrm{SMe}$ are plotted with those obtained with the BSSE eliminated to illustrate the unimportance of these effects in Figure 2. However, since the BSSE-corrected results were very similar to those obtained when BSSE were not removed, we decided to limit our discussion to the latter case.

These same procedures were followed with positive charges (simulating the two protonated amine sites discussed earlier) placed 10,20 , or $30 \AA$ from each of the disulfide bond's S atoms. The distance from each positive charge to the nearest $S$ atom was held fixed as the $\mathrm{S}-\mathrm{S}$ distance was scanned and the other geometrical degrees of freedom were optimized.

While carrying out the relaxed scans of the potential energy surface (PES) of $\mathrm{MeS}-\mathrm{SMe}^{-}$, we had to examine the PES region, where this anion is electronically unstable (for small $R$ values, where $R$ denotes the $\mathrm{S}-\mathrm{S}$ bond length). In such cases, we were able to achieve the proper electronic configuration of the anion by altering the virtual molecular orbitals of the neutral $\mathrm{MeS}-\mathrm{SMe}$ and forcing the SCF procedure to converge to the doublet anionic state in which the $\mathrm{S}-\mathrm{S}$ antibonding $\left(\sigma^{*}\right)$ orbital is singly occupied. In the absence of such care, the anion calculation would, as is well-known, undergo variational collapse and yield an energy essentially equal to that of the neutral plus a free electron very far away. These difficulties relate to the fact that the $\sigma^{*}$ anion is embedded in a continuum of other states. ${ }^{16}$ Even when employing such devices to properly characterize the metastable $\sigma^{*}$ anion, our computations are not able to yield an estimate of the autodetachment lifetime of this
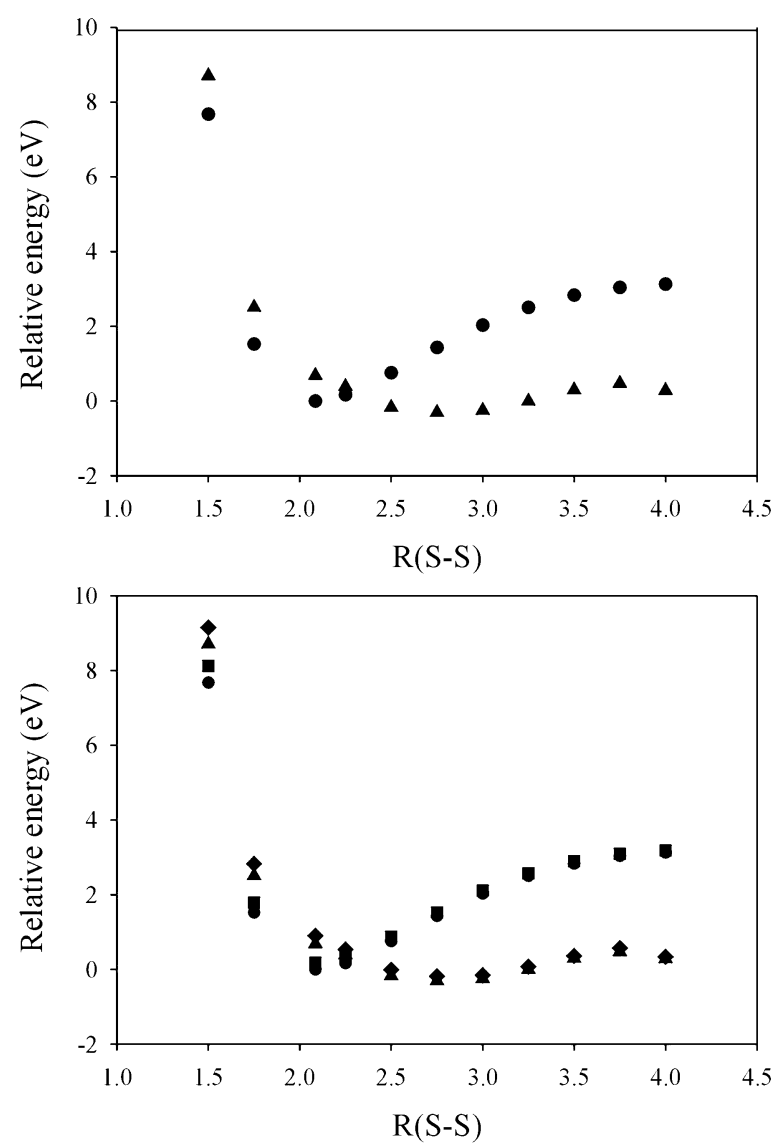

Figure 2. Variation (top) of the neutral (circles) and anion (triangles) energies (eV; relative to the neutral's minimum) along the $\mathrm{S}-\mathrm{S}$ bond length $(\AA)$ with all other geometrical parameters relaxed to minimize the neutral or anion energy. Comparison (bottom) of the BSSE-corrected results (squares for the neutral and diamonds for the anion) and uncorrected results (circles for the neutral and triangles for the anion).

anion state. However, these lifetimes are reasonably well-known and are not the focus of this work. Our focus is on determining at what $R$ value and what energy the neutral and anion surfaces cross and when the anion is electronically stable and when it is not.

The electronic instability of the $\mathrm{MeS}-\mathrm{SMe}^{-}$anion does not persist for larger (i.e., > $2.2 \AA$ ) $\mathrm{S}-\mathrm{S}$ bond lengths where the excess electron can be localized on either $\mathrm{S}-\mathrm{CH}_{3}$ fragment, which is known ${ }^{17}$ to possess a positive electron affinity of ca. $1.9 \mathrm{eV}$. Moreover, the electronic instability of $\mathrm{MeS}-\mathrm{SMe}^{-}$is an issue only in the absence of the additional positive stabilizing charges. As we show below, when these charges are present, the anionic energy curve is shifted down to lower energies where it remains "below" the corresponding neutral energy curve for any $\mathrm{S}-\mathrm{S}$ bond length. This is because the resonance anion (with the $\sigma^{*}$ orbital singly occupied) is stabilized by the electrostatic Coulomb potential of the positive point charges added.

The electrostatic potential maps shown later were generated with the MOLDEN program. ${ }^{18}$ All calculations were performed with the GAUSSIAN98 program $^{19}$ on AMD Athlon $950 \mathrm{MHz}$ and Pentium IV 2.0GHz computers, as well as on SGI and Compaq Sierra numerical servers.

\section{Results}

In Figure 2 we show how the neutral and anion electronic energies vary along the $\mathrm{S}-\mathrm{S}$ bond length with all other internal geometry variables relaxed to minimize the neutral or anion energy, respectively. This allows us to examine where the 


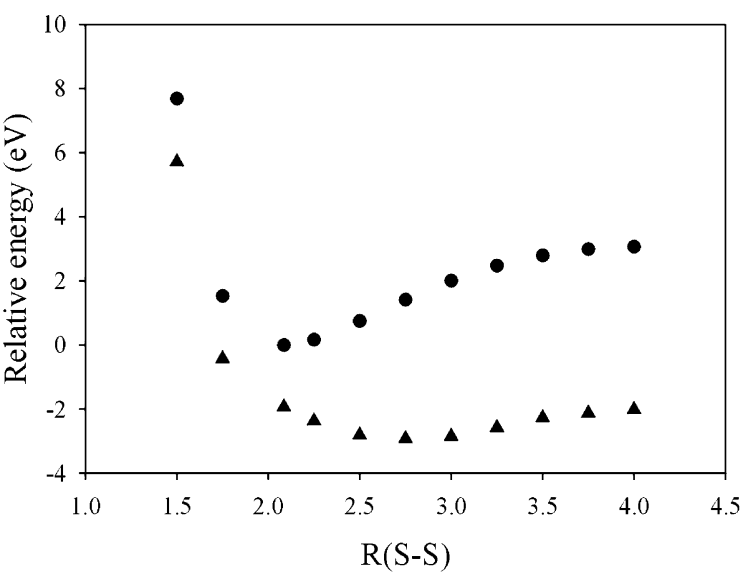

Figure 3. Neutral (circles) and $\sigma^{*}$ anion (triangles) energies $(\mathrm{eV})$ as functions of the $\mathrm{S}-\mathrm{S}$ bond length $(\AA)$ when two +1 charges are located $10 \AA$ from each $\mathrm{S}$ atom.

anion's energy surface lies relative to that of the neutral. This, of course, is of direct relevance for a process in which a free electron strikes the neutral molecule containing the $\mathrm{S}-\mathrm{S}$ bond and attaches to the $\sigma^{*}$ orbital of that bond.

The data used to construct Figure 2 allow us to make the following estimates:

(1) The dissociation energy for $\mathrm{MeS}-\mathrm{SMe} \rightarrow 2 \mathrm{MeS}$ is 2.2 $\mathrm{eV}$ or ca. $50 \mathrm{kcal} \mathrm{mol}^{-1}$;

(2) The electron affinity of MeS is $1.75 \mathrm{eV}$, a bit smaller than the experimental value ${ }^{17}$ of $1.9 \mathrm{eV}$;

(3) $R_{\mathrm{e}}(\mathrm{MeS}-\mathrm{SMe})=2.08 \AA$;

and $(4) \omega_{\mathrm{e}}(\mathrm{MeS}-\mathrm{SMe})=508 \mathrm{~cm}^{-1}$.

We note that the $\sigma^{*}$ anion lies $0.69 \mathrm{eV}$ above the minimum of the neutral species in these calculations, which is somewhat lower than the experimentally determined $1.04 \mathrm{eV}$ for the peak in the ETS spectrum (although this peak is $0.5 \mathrm{eV}$ broad, and recall that the peak in the dissociative electron attachment yield lies below $1 \mathrm{eV}$ ). The anion and neutral curves cross ca. 0.33 $\mathrm{eV}$ above the neutral's minimum; this corresponds to between the fourth and fifth excited vibrational state of the $\mathrm{S}-\mathrm{S}$ bond, which suggests that the $\mathrm{S}-\mathrm{S}$ bond would have to be considerably vibrationally excited to access the point where the curves cross. It is this energy requirement that likely produces a barrier to electron attachment in this case and that would preclude $S-S$ bonds from undergoing direct ECD cleavage in the absence of the stabilizing Coulomb potential that we discuss below. Another factor that makes the crossing point of the neutral and anion curves important is the fact that the anion is very short-lived (with respect to electron detachment) for $R$ values smaller than that at the crossing. That is, electrons can attach for bond lengths shorter than that of the crossing point, but the nascent anion will undergo autodetachment within ca. $10^{-14} \mathrm{~s}$. Finally, we also note that the anion surface has a shallow minimum lying $0.31 \mathrm{eV}$ below the minimum of the neutral surface, thus suggesting a positive adiabatic electron affinity that is a bit larger than the RET adiabatic electron affinity estimate mentioned earlier.

In Figure 3 we show the corresponding neutral and anion energies for the case in which two +1 charges have been located $10 \AA$ distant from each of the two sulfur atoms (and along the $\mathrm{S}-\mathrm{S}$ bond axis). The positive charges are used to represent the Coulomb potential presented by two protonated amine sites as, for example, in the dication shown in Figure 1.

We note that in Figure 3 the anion is electronically stable for all values of the $\mathrm{S}-\mathrm{S}$ bond length. As such, the neutral species is predicted to be able to attach an electron without

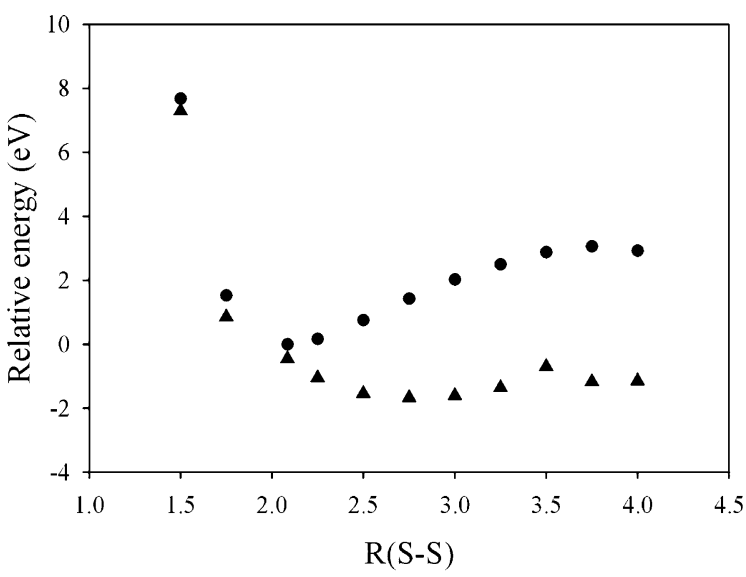

Figure 4. Neutral (circles) and $\sigma^{*}$ anion (triangles) energies $(\mathrm{eV})$ as functions of the $\mathrm{S}-\mathrm{S}$ bond length $(\AA)$ when two +1 charges are located $20 \AA$ from each $\mathrm{S}$ atom

having to overcome any barrier to access a curve crossing as in Figure 1. Note that the energy of the anion relative to the neutral at $R=2.08 \AA$ (the neutral's $R_{\mathrm{e}}$ ) moves from $0.69 \mathrm{eV}$ in Figure 2 to $-1.93 \mathrm{eV}$ in Figure 3. This $2.62 \mathrm{eV}$ stabilization is very close to that predicted by eq 1 for two positive charges at $10 \AA$ : $2(14.4 /(10+2.08 / 2)) \mathrm{eV}=2.6 \mathrm{eV}$.

This success of such a simple Coulomb stabilization model may be surprising, but we remind the reader that this same kind of model has proven highly successful ${ }^{20}$ when used to predict the electronic stabilities of multiply charged anions. In such cases, the vertical electron detachment energies can be computed reasonably accurately by reducing the intrinsic binding energy of a given anion site (i.e., its electron binding energy in the absence of any other negative sites) by the Coulomb repulsion energy produced by the other negative sites in the multiply charged anion.

Before examining the results obtained with the two +1 charges placed 20 or $30 \AA$ from each $\mathrm{S}$ atom, let us see what the Coulomb model would predict in those two cases. Specifically, it predicts the anion to be located relative to the minimum of the neutral by the following amounts:

$$
\begin{aligned}
0.69-2(14.4) /(21.04)= & -0.68 \mathrm{eV} \\
& \text { with the two }+1 \text { charges } 20 \AA \text { away } \\
0.69-2(14.4) /(31.04)= & -0.24 \mathrm{eV} \\
& \text { with the two charges } 30 \AA \text { away }
\end{aligned}
$$

So, in either case, we expect the $\mathrm{S}-\mathrm{S}$ bond to be able to attach an electron with no energy barrier.

In Figures 4 and 5 we show the neutral and $\sigma^{*}$ anion energies when two +1 charges are placed 20 and $30 \AA$ from the $\mathrm{S}$ atoms, respectively.

Clearly, even when the two protonated sites are $30 \AA$ away, the $\sigma^{*}$ disulfide anion is electronically stable with respect to the neutral near the equilibrium bond length of the neutral. So, direct electron attachment to the $\mathrm{S}-\mathrm{S}$ bond to effect fragmentation seems to be possible when two positive sites are present and as distant as $30 \AA$.

These findings and the Coulomb attraction potential model that seems to be consistent with them suggest that when the two protonated sites are further away than ca. $41 \AA$, the $\sigma_{\mathrm{SS}} *$ anion will not be vertically stable ${ }^{21}$ relative to the neutral, so direct electron attachment would then require energy input (e.g., vibrational excitation to access the surface crossing). Alternatively, these same data and the Coulomb model suggest that a 


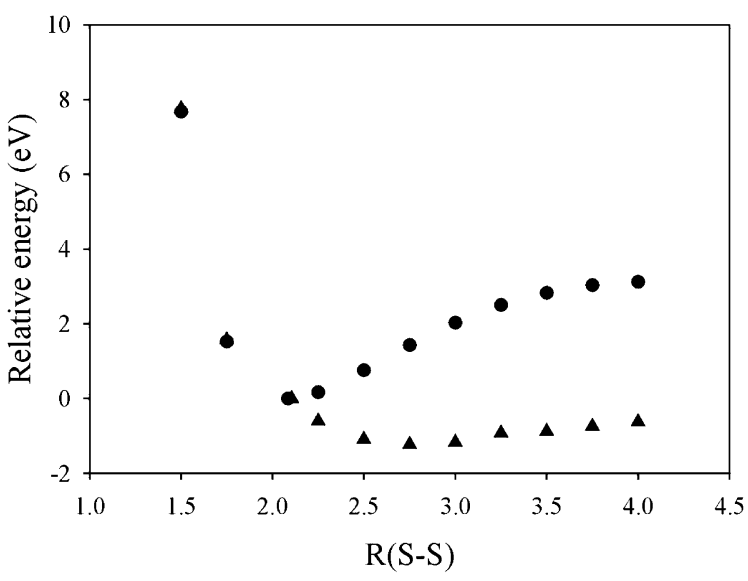

Figure 5. Neutral (circles) and $\sigma^{*}$ anion (triangles) energies $(\mathrm{eV})$ as functions of the $\mathrm{S}-\mathrm{S}$ bond length $(\AA)$ when two +1 charges are located $30 \AA$ from each $\mathrm{S}$ atom.

single protonated site $20 \AA$ distant or closer is adequate to stabilize the anion sufficiently to permit direct electron attachment. Single positively charged sites more distant than $20 \AA$ are not expected to permit direct attachment. These predictions should be subjected to further experimental tests.

Because the Coulomb potential plays a central role in the model outlined above, it is important to examine the full electrostatic potential experienced by an electron as it approaches the $S-S$ bond region both in the absence of any +1 charges and with such charges present. First, in Figure 6, we show the electrostatic potential for $\mathrm{MeS}-\mathrm{SMe}$ in the absence of any positive charges, with blue coloring denoting attractive regions and red coloring labeling repulsive regions.

Clearly, the regions where the lone-pair orbitals of the two sulfur atoms exist are repulsive while regions on the positive side of this molecule's dipole moment are attractive. Most importantly, there is no attractive region near the $\mathrm{S}-\mathrm{S}$ bond that would tend to guide an incident electron toward the SS $\sigma^{*}$ orbital.

In Figure 7 we show the electrostatic potential (with the same color convention) for four cases: with no +1 charges and with two +1 charges 30,20 , and $10 \AA$ from each sulfur atom.

These figures make it clear that an ECD electron is most strongly attracted to regions near the positively charges sites where deep potential wells clearly exist. Hence, it is likely that the mechanism described in Scheme 1 will attract the majority of the electrons, because these deep wells likely dominate the electron-capture process. However, it is also clear that other local minima in the potential energy surface experienced by such electrons exist in the regions of the $\mathrm{S}-\mathrm{S}$ bond when the positive charges are present at the three distances treated here. It is through the operation of such attractive potentials that we suggest a fraction of the ECD electrons enter the $\mathrm{S}-\mathrm{S} \sigma^{*}$ orbital and thus effect $\mathrm{S}-\mathrm{S}$ bond cleavage. It should be noted that in most electrospray experiments on proteins, the number of positively charged sites is usually large enough to produce the kind of stabilization discussed here. For example, a protein of mass $12 \mathrm{kDa}$ such as cytochrome $c$ typically has seven or eight positive charges when in a compact state and 15-20 charges when in an extended state.

\section{Summary}

Our results show that

(1) In the absence of Coulomb stabilization, direct vertical dissociative attachment of an electron to an $\mathrm{S}-\mathrm{S} \sigma^{*}$ orbital requires the electron to have a kinetic energy of ca. $0.5 \mathrm{eV}$ (experiments probing the position of the $\sigma^{*}$ shape resonance and the onset of dissociative electron attachment give somewhat

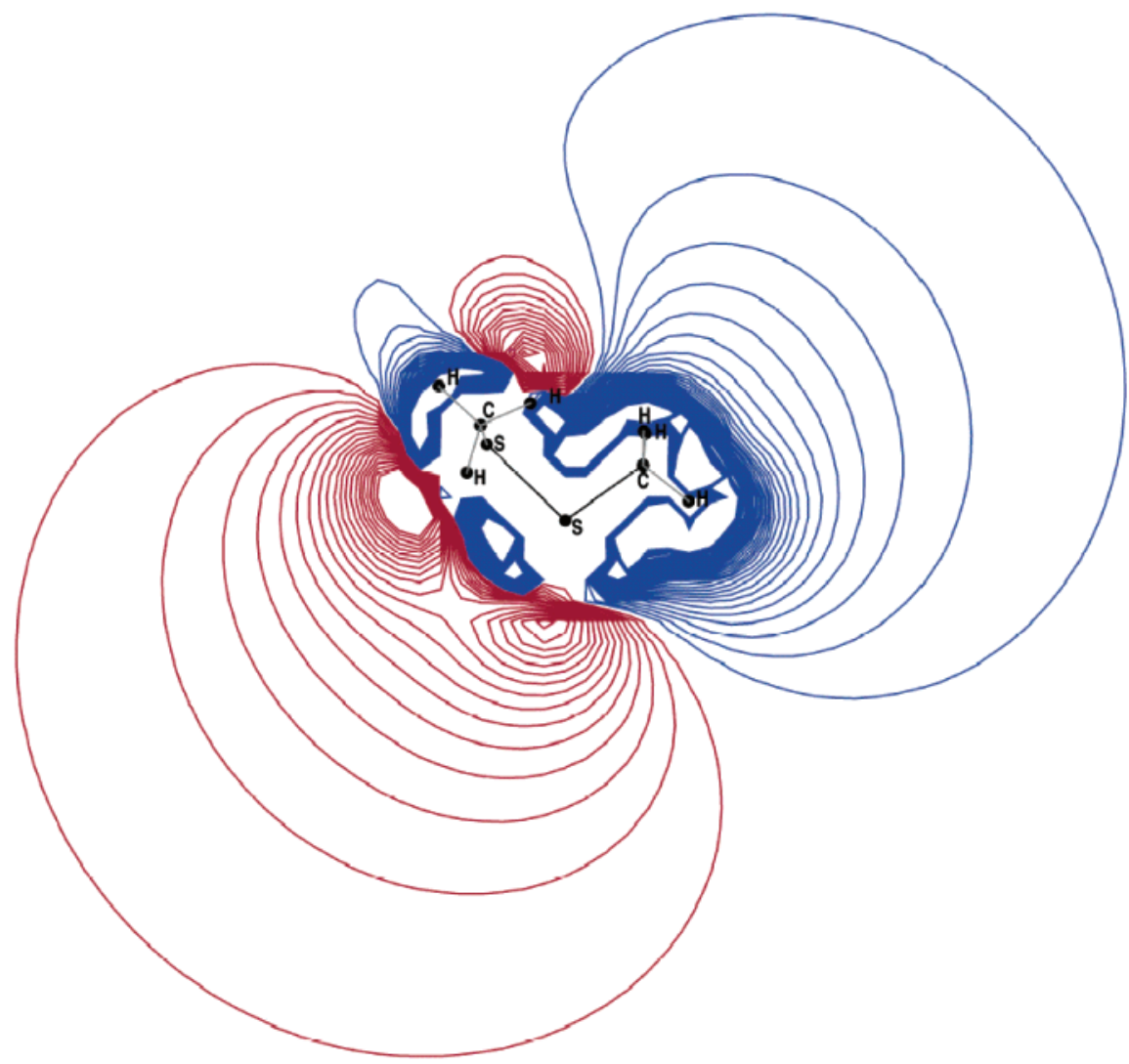

Figure 6. Electrostatic potential of $\mathrm{MeS}-\mathrm{SMe}$ with countours spaced by 0.0015 atomic units (i.e., $0.0408 \mathrm{eV}$ as an electron experiences the potential). Red regions are repulsive; blue are attractive. 

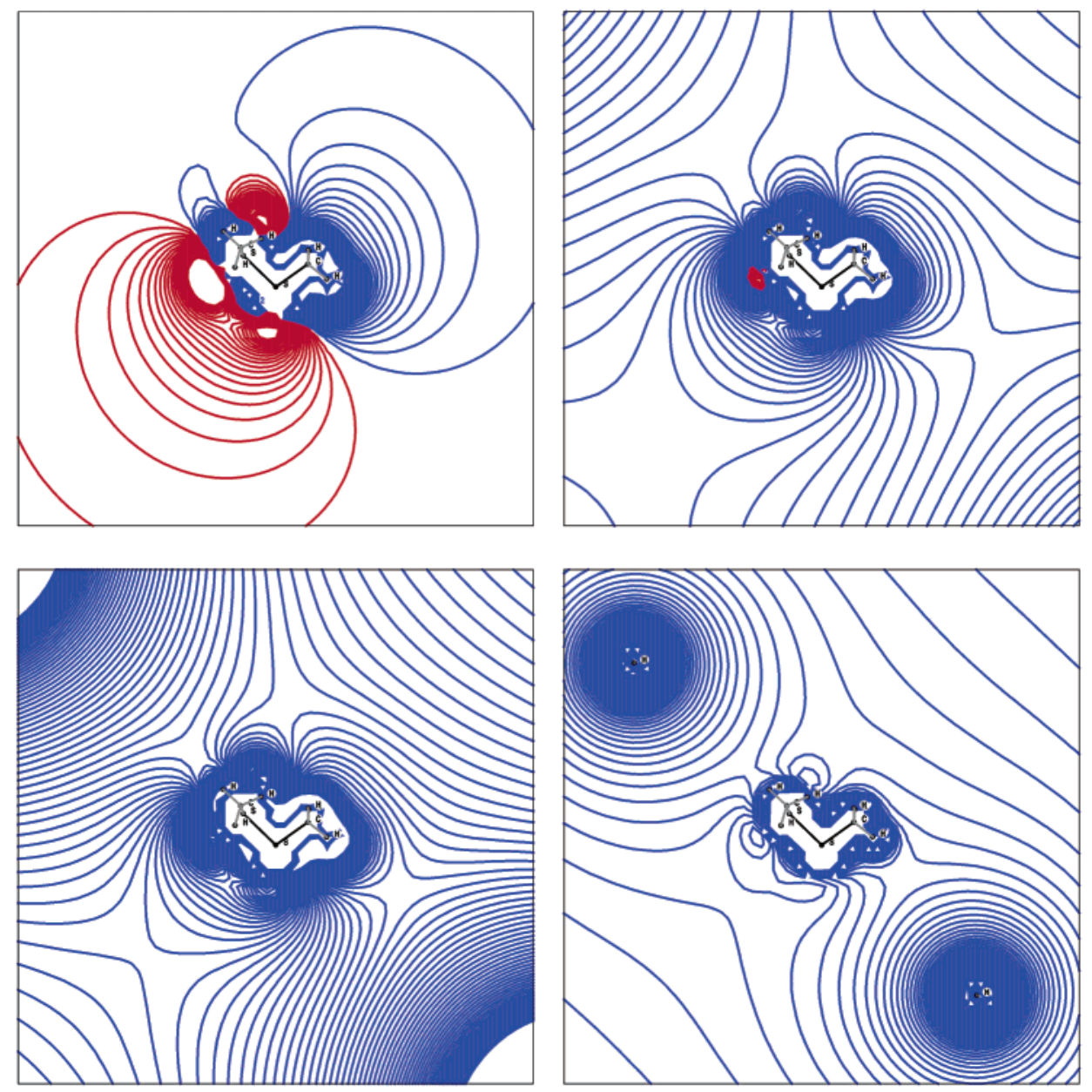

Figure 7. Electrostatic potentials showing attractive (blue) and repulsive (red) regions for MeS-SMe with no charges (upper left) and with two +1 charges 30,20 , and $10 \AA$ from each sulfur atom, respectively, clockwise from upper right.

higher values). Alternatively, substantial excitation of the $S-S$ vibration would be required to access the bond length at which the neutral and anion surfaces cross and thus could attach an even lower energy electron. These observations suggest that direct attachment of ECD electrons to $\mathrm{S}-\mathrm{S}$ bonds in the absence of any nearby positive sites is endothermic and very unlikely.

(2) In the presence of two stabilizing positive charges closer than ca. $41 \AA$ to the $S-S$ bond, the $\sigma^{*}$ anion surface of the disulfide is stabilized relative to the neutral surface to an extent that renders direct vertical electron attachment no longer endothermic.

(3) A single positive charge closer than $20 \AA$ should also allow direct dissociative attachment to occur with no energy requirement.

In addition to suggesting how Coulomb stabilization may alter a disulfide bond's ability to directly attach electrons in ECD experiments, we believe it is useful to speculate how such effects may play roles in rendering other bonds subject to cleavage via a direct-attachment mechanism. In particular, we note that $\mathrm{C}-\mathrm{O} \sigma^{*}$ and $\mathrm{C}-\mathrm{S} \sigma^{*}$ orbitals have been found in ETS experiments ${ }^{10}$ to lie vertically ca. $4.2 \mathrm{eV}$ and $<3.75 \mathrm{eV}$ above their corresponding neutrals. We suggest, therefore, that a single +1 site within $3.4 \AA$ would be needed to render $\mathrm{C}-\mathrm{O} \sigma$ bonds susceptible to direct-attachment ECD cleavage and a +1 site within $3.8 \AA$ could do likewise to a $\mathrm{C}-\mathrm{S} \sigma$ bond. These predictions should also be amenable to experimental verification.

If the suggestions provided in this paper are determined by further experimental testing to be true, it seems that ECD fragmentation patterns may need to be reexamined to take into consideration direct fragmentation of $\mathrm{S}-\mathrm{S}, \mathrm{C}-\mathrm{O}$, and $\mathrm{C}-\mathrm{S}$ (as well as $\mathrm{C}-\mathrm{C}$ ) $\sigma$ bonds in proximity to protonated sites. That is, fragmentation through the processes outlined in Schemes 1 and 2 are likely operative and may even dominate because the electrostatic potential causes most ECD electrons to be directed toward and to attach to the protonated sites. However, additional fragmentation via a direct-attachment mechanism such as that discussed in this paper may also be operative; if so, it is important to include such possibilities in interpreting fragmentation patterns.

Acknowledgment. This work has been supported by National Science Foundation Grants 9982420 and 0240387 to J.S. and by the Polish State Committee for Scientific Research (KBN) in Grant No. DS/8371-4-0137-3 to P.S.. The FT-ICR experimental work was supported in part by the NSF National High Field FT-ICR facility (CHE-99-09502).

\section{References and Notes}

(1) Zubarev, R.; Kruger, N.; Fridriksson, E. K.; Lewis, M. A.; Horn, D. M.; Carpenter, B. K.; McLafferty, F. W. J. Am. Chem. Soc. 1999, 121 , $2857-2862$.

(2) It is likely that the low-energy electron first attaches to a higher Rydberg orbital to form a very weakly bound electronic state, after which rapid radiationless relaxation leads to lower energy plus ground-state Rydberg species. Because the latter (i.e., $\mathrm{R}-\mathrm{NH}_{3}$ species) lie more than $3 \mathrm{eV}$ below the parent cation (i.e., $\mathrm{RNH}_{3}{ }^{+}$), it is highly improbable that the electron attaches to the cation to form the ground-state radical in a single step.

(3) It has also been shown that the $\mathrm{N}-\mathrm{C}$ bond can break to generate alternative fragmentation (see, for example, Shaffer, S. A.; Sadilek, M.; Turecek, F. J. Org. Chem. 1996, 61, 5234-5245). 
(4) Zubarev, R. A.; Horn, D. M.; Fridriksson, E. K.; Kelleher, N. L.; Kruger, N. A.; Lewis, M. A.; Carpenter, B. K.; McLafferty, F. W. Anal. Chem. 2000, 72, 563-573.

(5) Syrstad, E. A.; Turecek, F. J. Phys. Chem. 2001, A105, 111441115. Turecek, F.; Syrstad, E. A. J. Am. Chem. Soc. 2003, 125, 33533369.

(6) Turecek, F.; Polasek, M.; Frank, A.; Sadilek, M. J. Am. Chem. Soc. 2000, 122, 2361-2370.

(7) Although not yet published, these results have been presented in public: Hudgins, R. R.; Håkansson, K.; Quinn, J. P.; Hendrickson, C. L.; Marshall, A. G. Proceedings of the $50^{\text {th }}$ ASMS Conference on Mass Spectrometry and Allied Topics, Orlando, Florida, June 2-6, 2002.

(8) Hudgins, R. R.; Hakansson, K.; Quinn, J. P.; Hendrickson, C. L.; Marshall, A. G. Manuscript in preparation.

(9) It is thought that ECD sources contain electrons having a distribution of kinetic energies but with very few having energies near or above $1 \mathrm{eV}$.

(10) Dezarnaud-Dandine, C.; Bournel, F.; Tronc, M.; Jones, D.; Modelli, A. J. Phys. B. At. Mol. Opt. Phys. 1998, 31, L497-L501. However, it should also be mentioned that the yield of $\mathrm{RS}^{-}$anions observed in $\mathrm{e}^{-}+\mathrm{R}-\mathrm{S}-$ $\mathrm{S}-\mathrm{R}^{\prime}$ dissociative electron attachment experiments peaks at a somewhat lower energy (e.g, see: Modelli, A.; Jones, D.; Distefano, G.; Tronc, M. Chem. Phys. Lett. 1991, 181, 361-366). This indicates that for some values of the $\mathrm{S}-\mathrm{S}$ bond length the anion lies less than $1.04 \mathrm{eV}$ above the neutral.

(11) Carles, S.; Lecomte, F.; Schermann. J. P.; Defrancois, C.; Xu, S. Niles, J. M.; Bowen, K. H.; Berges, J.; Houee-Levin, C. J. Phys. Chem. 2001, A105, 5622-5626.

(12) In ref 3 an analogous process was examined in which an electron attaches to a phenyl ring whose $\pi^{*}$ orbitals have been lowered by attaching $\mathrm{F}$ atoms to the ring $\mathrm{C}$ atoms. This phenyl group was not directly attached to the protonated amine site, so electron attachment to the phenyl moiety generated such a zwitterion species.

(13) We have a great deal of experience studying electron-dipole interactions, and we are well aware of the difficulties involved in properly treating such issues. In particular, because of the $r^{-2}$ dependence of this potential ( $r$ being the distance to the electron), special care must be taken to preclude the attached electron from falling into this strongly attractive potential instead of into the $\mathrm{S}-\mathrm{S} \sigma^{*}$ orbital. These issues have caused us to defer the inclusion of dipolar potentials into our study of how Coulomb potentials can alter the probability of $\mathrm{S}-\mathrm{S}$ bond cleavage via direct electron capture.

(14) Kendall, R. A.; Dunning, T. H., Jr.; Harrison, R. J. J. Chem. Phys. 1992, 96, 6796. We did not want to use bases containing extremely diffuse functions, because we want to make sure that the attached electron cannot be "sucked" onto either of the two positively charged sites. By employing only conventional bases with modestly diffuse functions, we allow the attached electron to only enter the $\mathrm{S}-\mathrm{S} \sigma^{*}$ orbital
(15) Chalasinski, G.; Gutowski, M. Chem. Rev. 1988, 88, 943.

(16) We chose to use the MP2 procedure to examine the anion and neutral species because past experience has shown us that electron correlation is important to include when studying stable and metastable anions. When the SCF calculation is carried out on the neutral, the lowest unoccupied molecular orbital (LUMO) is most likely not a good approximation to the desired $\sigma^{*}$ orbital of the anion; rather, it is most likely a very diffuse continuum-like orbital appropriate to $\mathrm{Me}_{2} \mathrm{~S}_{2}+\mathrm{e}^{-}$. Therefore, we need to (by inspection) identify an orbital within the virtual orbital space of the neutral SCF calculation to use as our best approximation to the $\sigma^{*}$ orbital. By then altering the virtual orbitals such that this selected orbital is identified as the LUMO, we are able to carry out the SCF calculation (and the subsequent MP2 calculation) on the $\sigma^{*}$ anion. Had we chosen to employ a configuration interaction including single excitations (CIS) or complete active space self-consistent field (CASSCF) calculation, we still would have been faced with identifying the proper $\sigma^{*}$ solution to focus on. Moreover, the CIS treatment would not have included any electron correlation, which we decided we wanted.

(17) Rienstra-Kiracofe, J. C.; Tschumper, G. S.; Schaefer, H. F., III; Nandi, S.; Ellison, G. B. Atomic and Molecular Electron Affinities. Chem. Rev. 2002, 102, 231-282.

(18) Schaftenaar, G.; Noordik, J. H. MOLDEN: A pre- and postprocessing program for molecular and electronic structures. J. Comput.Aided Mol. Design 2000, 14, 123.

(19) Frisch, M. J.; Trucks, G. W.; Schlegel, H. B.; Scuseria, G. E.; Robb, M. A.; Cheeseman, J. R.; Zakrzewski, V. G.; Montgomery, J. A.; Stratmann, R. E., Jr.; Burant, J. C.; Dapprich, S.; Millam, J. M.; Daniels, A. D.; Kudin, K. N.; Strain, M. C.; Farkas, O.; Tomasi, J.; Barone, V.; Cossi, M.; Cammi, R.; Mennucci, B.; Pomelli, C.; Adamo, C.; Clifford, S.; Ochterski, J.; Petersson, G. A.; Ayala, P. Y.; Cui, Q; Morokuma, K.; Malick, D. K. Rabuck, A. D.; Raghavachari, K.; Foresman, J. B.; Cioslowski, J.; Ortiz, J. V.; Baboul, A. G.; Stefanov, B. B.; Liu, G.; Liashenko, A.; Piskorz, P.; Komaromi, I.; Gomperts, R.; Martin, R. L.; Fox, D. J.; Keith, T.; Al-Laham, M. A.; Peng, C. Y.; Nanayakkara, A.; Gonzalez, C.; Challacombe, M.; Gill, P. M. W.; Johnson, B.; Chen, W.; Wong, M. W.; Andres, J. L.; Gonzalez, C.; Head-Gordon, M.; Replogle, E. S.; Pople, J. A. GAUSSIAN 98, Revision A.7; Gaussian, Inc., Pittsburgh, PA, 1998.

(20) Simons, J.; Skurski, P.; Barrios, R. J. Am. Chem. Soc. 2000, 122, 11893. Whitehead, A.; Barrios, R.; Simons, J. J. Chem. Phys. 2002, 116, 2848. Simons, J.; Skurski, P. Recent Reseach and Development in Physical Chemistry, Theoretical Prospect of Negative Ions; Kalcher, J., Ed., 2002; pp 117-138.

(21) We obtain this estimate by equating the intrinsic instability of the anion $(0.69 \mathrm{eV})$ to the Coulomb model's prediction of stabilization 2(14.4)/ $(R+2.08 / 2)$ in eV 